TRANSACTIONS OF THE

AMERICAN MATHEMATICAL SOCIETY

Volume 350, Number 11, November 1998, Pages 4633-4649

S 0002-9947(98)01992-8

\title{
SUBELLIPTIC HARMONIC MAPS
}

\author{
JÜRGEN JOST AND CHAO-JIANG XU
}

\begin{abstract}
We study a nonlinear harmonic map type system of subelliptic PDE. In particular, we solve the Dirichlet problem with image contained in a convex ball.
\end{abstract}

\section{INTRODUCTION}

Building upon and extending the basic work of Hörmander $[\mathrm{H}]$, a general theory of subelliptic PDE has been developed in the work of several authors (see e.g. [B], $[\mathrm{FP}],[\mathrm{Je}],[\mathrm{JeS}],[\mathrm{NSW}],[\mathrm{RS}],[\mathrm{S}],[\mathrm{X} 1],[\mathrm{X} 2]$ and many others), and it has been found that many important results carry over from the elliptic to the hypoelliptic case. It therefore seems natural to try to extend also the recent advances in elliptic PDE concerned with quasilinear and nonlinear problems of a variational origin to the hypoelliptic case. The present paper aims to take a first step in this direction and to establish some foundations by investigating in detail a variational problem that turned out to be a model problem for the elliptic case, namely the harmonic map problem, in a hypoelliptic setting. One of the important features of the harmonic map problem is its geometric nature, so that geometric intuition can guide the development of analytic tools. From a geometric point of view, however, hypoelliptic operators lead to a highly singular domain metric. Fortunately, we have here the recent work of the first author ([J2], [J3], [J4]) at our disposal; he constructed a variational harmonic map theory for very general singular domains and also dissociated the underlying integration measure from the domain metric, which is required by the present context as well.

After the original work of Eells-Sampson [ES], Al'ber [A1], [A2] and Hamilton $[\mathrm{H}]$ on harmonic maps with values in Riemannian manifolds of nonpositive curvature that employed parabolic methods, Hildebrandt-Kaul-Widman [HKW] in a fundamental paper introduced variational methods that also allowed a treatment of positive image curvature. Here, however, one meets a principal limitation of the harmonic map theory, because Hildebrandt-Kaul-Widman discovered that harmonic maps may develop singularities unless their range satisfies a certain convexity condition. They then established an existence and regularity result under this convexity condition.

In the present paper, we first collect some results about hypoelliptic PDE from the literature, in particular about the behavior of the associated Green functions. We then use the constructions of $[\mathrm{J} 1],[\mathrm{J} 2]$ to define an energy integral which we then

Received by the editors November 10, 1995 and, in revised form, November 10, 1996.

1991 Mathematics Subject Classification. Primary 35H05, 58E20, 35J20.

The essential part of the present work was carried out when the second author was a guest of the research project SFB 237 at the Ruhr University Bochum. The first author was also supported by the DFG, the second one by the NSF of China. 
attempt to minimize. The main result of our paper is an existence and regularity theorem for harmonic maps under the convexity condition of [HKW] on the image. The proof will in particular depend on precise Green function estimates. The somewhat complicated iteration technique of $[\mathrm{HKW}]$ and $[\mathrm{HW}]$ will be avoided by utilizing a more geometric construction of Kendall [K].

In order to place the present work into a proper perspective, apart from the relations to hypoelliptic PDEs, we should also mention the relation to the regularity aspects of generalized harmonic maps as further explored in [J5]. Finally, we hope that this direction of research will also be useful for questions on the rigidity of group representations, like discrete groups acting on spaces that carry a natural Hörmander type geometry.

We shall employ standard summation conventions.

\section{THE GEOMETRY DEFINED BY VECTOR FIELDS SATISFYING HÖRMANDER'S CONDITION}

Let $\Omega$ be an open domain in $\mathbb{R}^{n}, n \geq 2$. Let $X_{1}, \ldots, X_{m}$ be a system of vector fields with smooth real coefficients defined on some $\tilde{\Omega}$ with $\Omega \subset \subset \tilde{\Omega}$. The essential hypothesis is Hörmander's condition:

$(\mathbf{H}): X_{1}, \ldots, X_{m}$ together with their commutators up to a certain fixed length $r$ span the tangent space at each point of $\tilde{\Omega}$.

For $\Omega$, we suppose that $\partial \Omega$ is smooth and noncharacteristic for the system $X_{1}, \ldots$, $X_{m}$, i.e. $\forall x_{0} \in \partial \Omega \exists j_{0}$ such that $X_{j_{0}}\left(x_{0}\right) \notin T_{x_{0}} \partial \Omega$. In general, our system of vector fields is degenerate elliptic.

Example. $X_{1}=\partial_{x_{1}}, X_{2}=\partial_{x_{2}}+x_{1}^{k} \partial_{x_{3}}, k \geq 1$, is a Hörmander type system on $\mathbb{R}^{3}$ with $r=k+1$.

For abbreviation, we shall occasionally write $X:=\left(X_{1}, \ldots, X_{m}\right)$. From this system of vector fields, we can define a non-isotropic metric in the following way. For $0<\delta<1$, we say $\varphi:[0,1] \rightarrow \tilde{\Omega}$ belongs to $C(\delta)$ if $\varphi$ is Lipschitz and

$$
\dot{\varphi}(t)=\sum_{j=1}^{m} \alpha_{j}(t) X_{j}(\varphi(t)), \quad \text { a.e. } t \in[0,1],
$$

with $\left|\alpha_{j}(t)\right|<\delta$. We define for $x, y \in \Omega,|x-y| \ll 1$,

$$
\varrho(x, y):=\inf \{\delta, \exists \varphi \in C(\delta), \varphi(0)=x, \varphi(1)=y\} .
$$

The Hörmander condition implies that

$$
\begin{gathered}
\varrho(x, y) \leq \varrho(x, z)+\varrho(z, y) \quad \forall x, y, z \in \Omega, \\
C_{1}|x-y| \leq \varrho(x, y) \leq C_{2}|x-y|^{\frac{1}{r}}
\end{gathered}
$$

for all $x, y \in \tilde{\Omega},|x-y| \ll 1$ (see [Je], [JeS]). We write $X_{j}=b^{j k} \partial_{x^{k}}$; the adjoint of $X_{j}$ then is defined by $X_{j}^{*} f=-\partial_{x^{k}}\left(b^{j k} f\right)$ for $f \in C_{0}^{1}(\tilde{\Omega})$. We consider the Hörmander operator

$$
H=\sum_{j=1}^{m} X_{j}^{*} X_{j}=-\sum_{i, k=1}^{m} \partial_{x^{i}}\left(a^{i k}(x) \partial_{x^{k}}\right)
$$

with $a^{i k}(x)=b^{j i}(x) b^{j k}(x)$. In particular, $a^{i k} \in C^{\infty}(\tilde{\Omega})$ and $\left(a^{i k}(x)\right)_{i, k=1, \ldots, m}$ is symmetric and positive semidefinite. It need not be positive definite, however, and 
therefore, in general, $H$ is not elliptic. We approximate $H$ by the second order elliptic operator

$$
H_{\varepsilon}=H-\varepsilon \Delta \quad(\varepsilon>0) .
$$

These operators are Laplace type operators associated with the Riemannian metrics

$$
\left(a_{i k}^{\varepsilon}(x)\right)=\left(a^{i k}(x)+\varepsilon \delta_{i k}\right)^{-1}
$$

on $\tilde{\Omega}$. For the associated distance functions $d_{\varepsilon}(\cdot, \cdot)$, we have (see $[\mathrm{S}]$ )

$$
d_{\varepsilon}(x, y) \leq d_{\varepsilon^{\prime}}(x, y) \quad \text { for } 0<\varepsilon^{\prime} \leq \varepsilon
$$

and

$$
\lim _{\varepsilon \rightarrow 0} d_{\varepsilon}(x, y)=\varrho(x, y) .
$$

For the associate balls $B_{H}(x, \delta)=\{y ; \varrho(x, y)<\delta\}$, we have (see [Je])

$$
\left|B_{H}(x, 2 \delta)\right| \leq C\left|B_{H}(x, \delta)\right| .
$$

$(\Omega, \varrho)$ is some kind of degenerate Riemannian manifold because the distance balls satisfy

$$
B_{H}(x, \delta)=\bigcap_{\varepsilon>0} B_{H_{\varepsilon}}(x, \delta) .
$$

In the theory of subelliptic PDE, such an $(\Omega, \varrho)$ is called a homogeneous space.

We shall also need the following construction: From the above description of $\varrho$, one concludes that

$$
\left|X_{j} \varrho\right| \leq 1 \text { for all } j .
$$

If now $0<R_{1}<R_{2}$ and $B_{H}\left(x, R_{2}\right) \subset \Omega$, we put

$$
\eta(y):= \begin{cases}1 & \text { for } y \in B_{H}\left(x, R_{1}\right) \\ 0 & \text { for } y \in \Omega \backslash B_{H}\left(x, R_{2}\right) \\ \frac{R_{2}-\varrho(x, y)}{R_{2}-R_{1}} & \text { for } y \in B_{H}\left(x, R_{2}\right) \backslash B_{H}\left(x, R_{1}\right) .\end{cases}
$$

Then $\eta$ is a Lipschitz function with $0 \leq \eta \leq 1$, and

$$
\left|X_{j} \eta\right| \leq \frac{1}{R_{2}-R_{1}} \quad \text { for all } j \quad \text { by }(2.6) .
$$

\section{FunCtion SPACES ASSOCiated With VECTOR FIELDS}

In order to study weak solutions, we introduce a function space $M^{k}(\Omega)$ associated with $X$, which is an analogue of the usual Sobolev space. For $k \geq 1$, we define

$$
M^{k}(\Omega):=\left\{f \in L^{2}(\Omega) ; X^{J} f \in L^{2}(\Omega), \quad \forall|J| \leq k\right\},
$$

where $J=\left(j_{1}, \ldots, j_{s}\right), 1 \leq j_{l} \leq m,|J|=s, X^{J} f=X_{j_{1}} \ldots X_{j_{s}} f$. This weighted Sobolev space $M^{k}(\Omega)$ is a separable Hilbert space. We denote by $M_{0}^{k}(\Omega)$ the closure of $C_{0}^{\infty}(\Omega)$ in $M^{k}(\Omega)$. From Hörmander's condition, we have

$$
M_{0}^{k}(\Omega) \subset H^{\frac{k}{r}}(\Omega) .
$$

We also define Hölder spaces by

$$
S^{0, \alpha}(\Omega):=\left\{u \in L^{\infty}(\Omega) ;[u]_{X}^{\alpha}=\sup _{x, y \in \Omega} \frac{|u(x)-u(y)|}{\varrho(x, y)^{\alpha}}<+\infty\right\},
$$




$$
S^{k, \alpha}(\Omega):=\left\{u \in S^{\alpha}(\Omega) ; X^{J} \in S^{k}(\Omega), \quad \forall|J| \leq k\right\} .
$$

These non-isotropic Hölder spaces are Banach spaces and satisfy

$$
S^{k r, \alpha}(\Omega) \subset C^{k, \frac{\alpha}{r}}(\Omega) .
$$

We usually write $S^{k}$ instead of $S^{k, 0}$. These function spaces are well adapted to the operator $H$. Using the degenerate Riemannian structure of $\S 2$, and the present function spaces, the Hörmander operator $H$ enjoys many Laplacian type properties. We collect here those results that we shall exploit in the sequel.

Lemma 3.1 (Maximum principle of Bony [B]). Let $u \in S^{1, \alpha}(\Omega)$ satisfy $H u \leq 0$, $\alpha>0$. If there exists $x^{0} \in \Omega$ with $0 \leq u\left(x^{0}\right)=\sup _{\Omega} u(x)<+\infty$, then $u \equiv u\left(x^{0}\right)$ on $\Omega$. In fact, the maximum principle also holds for weak solutions $u \in M^{1}(\Omega)$ of $H u \leq 0$ in the usual sense.

Since the operator $H$ is hypoelliptic, the maximum principle immediately yields the uniqueness of (weak) solutions for the Dirichlet problem on $\Omega$.

Lemma 3.2. $\quad$ (i) (First Poincaré inequality on $\Omega$ )

$$
\|\varphi\|_{L^{2}(\Omega)} \leq C(\Omega)\|X \varphi\|_{L^{2}(\Omega)}, \quad \forall \varphi \in M_{0}^{1}(\Omega) .
$$

(ii) (First Poincaré inequality on $B_{H}\left(x_{0}, R\right)$ for $R$ small enough). $\forall x_{0} \in \Omega \exists R_{0}>0, C\left(R_{0}\right)>0$ such that $\forall 0<R \leq R_{0}$

$$
\|\varphi\|_{L^{2}\left(B_{H}\left(x_{0}, R\right)\right)} \leq C\left(R_{0}\right) R\|X \varphi\|_{L^{2}\left(B_{H}\left(x_{0}, R\right)\right)}, \quad \forall \varphi \in M_{0}^{1}\left(B_{H}\left(x_{0}, R\right)\right)
$$

(iii) $\forall x_{0} \in \Omega \exists R_{0}>0$ with $B_{H}\left(x_{0}, 2 R_{0}\right) \subset \Omega, C\left(R_{0}\right)>0$, such that for $0<R \leq$ $R_{0}$

$$
\int_{B_{H}\left(x_{0}, R\right)}\left|u-\bar{u}_{R}\right|^{2} \leq C\left(R_{0}\right) R^{2} \int_{B_{H}\left(x_{0}, R\right)}|X u|^{2}
$$

$\forall u \in M^{1}\left(B_{H}\left(x_{0}, R\right)\right)$, where

$$
\bar{u}_{R}=\left|B_{H}\left(x_{0}, R\right)\right|^{-1} \int_{B_{H}\left(x_{0}, R\right)} u(x) d x .
$$

Proof. (i) follows from the strong maximum principle of Lemma 3.1.

(ii) For $x^{0} \in \Omega$, Hörmander's condition implies that there exists $j_{0}$ such that $X_{j_{0}}\left(x_{0}\right) \neq 0$. We change the variables so that $X_{j_{0}}=\partial_{x_{k}}$ in $B_{H}\left(x_{0}, R\right) \subset B_{\Delta}\left(x_{0}, R\right)$. The classical Poincaré inequality then gives the results.

(iii) is a result of Jerison [Je]. We remark that, using the proof of [Je], we can also obtain

$$
\int_{T_{R}}\left|u-\overline{\bar{u}}_{R}\right|^{2} \leq C R^{2} \int_{T_{R}}|X u|^{2}
$$

where $T_{R}=B_{H}\left(x_{0}, R\right) \backslash B_{H}\left(x_{0}, \frac{1}{2} R\right)$ and $\overline{\bar{u}}_{R}=\left|T_{R}\right|^{-1} \int_{T_{R}} u(x) d x$.

q.e.d.

The hypoellipticity of the operator $H$, and the maximum principle imply that there exists the Green function $G(x, y)$ of $H$ on $\Omega$. We have

Lemma 3.3 (see [NSW], [S]). $\forall K \subset \subset \Omega$, we have $\forall x, y \in K$,

$$
\begin{gathered}
0<G(x, y) \leq C_{K} \varrho(x, y)^{2}\left|B_{H}(x, \varrho(x, y))\right|^{-1}, \\
\left|X^{J} G(x, y)\right| \leq C_{J, K} \varrho(x, y)^{2-|J|}\left|B_{H}(x, \varrho(x, y))\right|^{-1},
\end{gathered}
$$




$$
\varrho(x, y)^{2}\left|B_{H}(x, \varrho(x, y))\right|^{-1} \leq C_{K} G(x, y) \quad \text { for } \varrho(x, y) \leq \delta .
$$

Associated with this metric we have also

Lemma 3.4 (see [X2]). Let $K \subset \subset \Omega$ and put $K_{\delta}:=\{y \in \Omega, \varrho(x, y)<\delta, x \in K\}$. Then, for $0<\delta \leq \delta_{0}$ small enough $\exists \varphi_{\delta} \in C_{0}^{\infty}\left(K_{\delta}\right)$ such that $\varphi_{\delta}(x)=1, x \in K$, and $\left|X^{J} \varphi_{\delta}\right| \leq C_{J} / \delta^{|J|}$, where $C_{J}$ is independent of $\delta$.

Lemma 3.5 (see [X2]). For all $\alpha>0$ and $0<R \leq R_{0}$,

$$
\int_{B_{H}(x, R)} \varrho(x, y)^{\alpha}\left|B_{H}(x, \varrho(x, y))\right|^{-1} d y \leq C_{R_{0}, \alpha} R^{\alpha} .
$$

Using these results, we can obtain the optimal interior regularity results for the operator $H$.

Lemma 3.6 (see $[\mathrm{RS}]$, [X2]). Suppose that $H u=f$. Then, if $f \in M^{k}(\Omega)$, we have $u \in M^{k+2}\left(\Omega^{\prime}\right)$, and if $f \in S^{k, \alpha}(\Omega)$, we have $u \in S^{k+2, \alpha}\left(\Omega^{\prime}\right)$ for any $k \in \mathbb{N}, \alpha>0$, and all $\Omega^{\prime} \subset \subset \Omega$. We also have the a priori estimates

$$
\begin{aligned}
\|\varphi u\|_{M^{k+2}\left(\Omega^{\prime}\right)} & \leq C\left(\Omega^{\prime}\right)\left\{\left\|\varphi_{1} H u\right\|_{M^{k}(\Omega)}+\left\|\varphi_{1} u\right\|_{L^{2}(\Omega)}\right\}, \\
\|\varphi u\|_{S^{k+2, \alpha}\left(\Omega^{\prime}\right)} & \leq C\left(\Omega^{\prime}\right)\left\{\left\|\varphi_{1} H u\right\|_{S^{k, \alpha}(\Omega)}+\left\|\varphi_{1} u\right\|_{L^{\infty}(\Omega)}\right\},
\end{aligned}
$$

for all $\varphi \in C_{0}^{\infty}\left(\Omega^{\prime}\right)$, where $\varphi_{1} \in C_{0}^{\infty}(\Omega), \varphi_{1}(x)=1, x \in \Omega^{\prime}$.

In the sequel, we shall also need some further Green function estimates which we now collect. They are similar to the estimates of Biroli-Mosco [BM], but for completeness, we provide proofs.

Lemma 3.7. Let $G(x, y)$ be the Green function of $H$ on $\Omega$. For $\sigma>0$ small enough, we define the mollified Green function by

$$
G^{\sigma}(x, y)=f_{B(y, \sigma)} G(x, z) d z .
$$

Then, $G^{\sigma}(\cdot, y) \in M_{0}^{1} \cap L^{\infty}(\Omega, R)$, and

$$
0 \leq G^{\sigma}(x, y) \leq C\left\{\begin{array}{l}
\varrho(x, y)^{2}|B(x, \varrho(x, y))|^{-1}, \quad \text { if } \sigma<c_{1} \varrho(x, y) \\
\sigma^{2}|B(x, \sigma)|^{-1}, \quad \text { if } \sigma \geq c_{1} \varrho(x, y),
\end{array}\right.
$$

and

$$
\left|X^{x} G^{\sigma}(x, y)\right| \leq C\left\{\begin{array}{l}
\varrho(x, y)\left|B_{H}(x, \varrho(x, y))\right|^{-1}, \quad \text { if } \sigma<c_{1} \varrho(x, y), \\
\sigma\left|B_{H}(x, \sigma)\right|^{-1}, \quad \text { if } \sigma \geq c_{1} \varrho(x, y),
\end{array}\right.
$$

and $\forall \xi \in M_{0}^{1}(\Omega)$, we have, if $B(y, \sigma) \subset \Omega$,

$$
\int_{\Omega} X \xi G^{\sigma}(\cdot, y)=f_{B(y, \sigma)} \xi(x) d x
$$

and $\lim _{\sigma \rightarrow \infty} G^{\sigma}(x, y)=G(x, y)$.

Proof. (3.19) is evident. Using Lemma 3.3 and (2.1), (2.4), we get (3.17) and

$$
\begin{aligned}
\left|X^{x} G^{\sigma}(x, y)\right| & =\left|f_{B_{\sigma}(y)} X^{x} G(x, z) d z\right| \\
& \leq C \begin{cases}\varrho(x, y)\left|B_{H}(x, \varrho(x, y))\right|^{-1}, & \sigma \leq c_{1} \varrho(x, y), \\
\delta\left|B_{H}(x, \delta)\right|^{-1}, & \sigma \geq c_{1} \varrho(x, y) .\end{cases}
\end{aligned}
$$


We have also proved $G^{\sigma}(\cdot, y) \in S^{1, \alpha}(\bar{\Omega}) \forall 0<\alpha<1$.

q.e.d.

Corollary 3.1. If $G(x, y)$ is the Green function of $H$ on $\Omega$, then

$$
0 \leq G(x, y) \leq C \varrho(x, y)^{2}|B(x, \varrho(x, y))|^{-1} \quad \forall x, y \in \bar{\Omega} .
$$

Proof. Denote by $\tilde{G}(x, y)$ the Green function of $H$ on $\tilde{\Omega} \supset \supset \Omega$. Then $\tilde{G}^{\sigma}(\cdot, y) \in$ $S^{1, \alpha}(\bar{\Omega})$. We now solve the following Dirichlet problem:

$$
\left\{\begin{array}{l}
H U_{y}^{\sigma}=0 \\
\left.U_{y}^{\sigma}\right|_{\partial \Omega}=\tilde{G}^{\sigma}(\cdot, y) \in C^{0}(\partial \Omega) .
\end{array}\right.
$$

Then the theorem of Bony [B] gives the existence of a solution $U_{y}^{\sigma} \in C^{0}(\bar{\Omega})$. Set $G_{y}^{\sigma}(x)=U_{y}^{\sigma}(x)+\tilde{G}^{\sigma}(x, y)$; then $H\left(G_{y}^{\sigma}-\tilde{G}^{\sigma}(\cdot, y)\right)=0$, and the maximum principle implies

$$
G_{y}^{\sigma}(x) \leq \tilde{G}^{\sigma}(x, y) \quad \forall x, y \in \bar{\Omega} .
$$

(3.20) now follows by letting $\sigma \rightarrow 0$.

q.e.d.

\section{Dirichlet And EnERgy FunCtionals ASSOCIATED WITH A HÖRMANDER OPERATOR}

Let $N$ be a complete Riemannian manifold without boundary of dimension $\geq 2$. We suppose that $N$ can be covered by a single coordinate chart, so that we can define the weighted Sobolev space $M^{1}(\Omega, N)$ unambiguously with the help of this chart, i.e.

$$
f \in M^{1}(\Omega, N) \Leftrightarrow \psi \circ f \in M^{1}\left(\Omega, \mathbb{R}^{\nu}\right),
$$

where $\psi: Y \rightarrow \mathbb{R}^{\nu}$ is a coordinate chart of class $C^{\infty}$. Similarly, we define the spaces $M_{0}^{1}(\Omega, N)$ and $S^{k, \alpha}(\Omega, N)$. For $p \in N$ and $r>0$, we put $B(p, r)=\{q \in N$ : $\left.d_{N}(p, q) \leq r\right\}$, where the distance function $d_{N}$ comes from the Riemannian metric. In intrinsic notation, the metric of $N$ is denoted by $\langle\cdot, \cdot\rangle_{N}$. In local coordinates the coefficients of the metric of $N$ are denoted by $\left(g_{\alpha \beta}(x)\right)$, with $\left(g^{\alpha \beta}(x)\right)$ being the inverse of $\left(g_{\alpha \beta}\right)$, while $\Gamma_{\beta \gamma}^{\alpha}$ are the Christoffel symbols.

We consider maps

$$
f:\left(\bar{\Omega}, d_{\varepsilon}\right) \rightarrow\left(N, d_{N}\right) .
$$

Since $\left(\bar{\Omega}, d_{\varepsilon}\right),\left(N, d_{N}\right)$ are Riemannian manifolds, with metric tensors $\left(a_{i j}^{\varepsilon}\right)$ and $\left(g_{\alpha \beta}\right)$, if $f: \bar{\Omega} \rightarrow N$ is a $C^{1}$ map, we can define the energy density

$$
\begin{aligned}
e_{\varepsilon}(f) & =\frac{1}{2}\left(a^{i j}(x)+\varepsilon \delta_{i j}\right) g_{\alpha \beta}(f) \frac{\partial f^{\alpha}}{\partial x_{i}} \frac{\partial f^{\beta}}{\partial x_{j}} \\
& =\frac{1}{2} g_{\alpha \beta}(f) X_{j} f^{\alpha} X_{j} f^{\beta}+\frac{\varepsilon}{2} g_{\alpha \beta}(f) \frac{\partial f^{\alpha}}{\partial x_{j}} \frac{\partial f^{\beta}}{\partial x_{j}} .
\end{aligned}
$$

Then the energy of $f$ is simply

$$
E_{\varepsilon}(f)=\int_{\Omega} e_{\varepsilon}(f) d \Omega
$$

with $d \Omega=d x$, the Lebesgue measure. For $f \in H^{1}(\Omega, N)$,

$$
\lim _{\varepsilon \rightarrow 0} E_{\varepsilon}(f)=\int_{\Omega} e(f) d x,
$$


where

$$
e(f)=\frac{1}{2} g_{\alpha \beta}(f) X_{j} f^{\alpha} X_{j} f^{\beta} .
$$

So we define the energy of a map $f:(\bar{\Omega}, \varrho) \rightarrow\left(N, d_{N}\right)$ by

$$
E(f)=\int_{\Omega} e(f) d x
$$

The Euler-Lagrange equation for this energy functional can be written in the form

$$
H u^{\alpha}+\Gamma_{\beta \gamma}^{\alpha}(u) X_{j} u^{\beta} X_{j} u^{\gamma}=0, \quad 1 \leq \alpha \leq \nu .
$$

We abbreviate (4.6) as $H_{N} u=0$ (thus $H_{\mathbb{R}}=H$ ).

We can now state our existence result for a solution of the Dirichlet problem for (4.6):

Main Theorem. Let $\kappa^{2}$ be an upper bound for the sectional curvature of $N$, and suppose that $p \in N$ and $\mu<\min (\pi / 2 \kappa, i(p))$, where $i(p)$ is the injectivity radius of $p \in N$. Let $\varphi \in C^{0}(\bar{\Omega}, N) \cap M^{1}(\Omega, N)$ satisfy

$$
\varphi(\bar{\Omega}) \subset B(p, \mu) .
$$

Then there exists a unique map $u \in M^{1}(\Omega, N) \cap L^{\infty}(\Omega, N)$ with $\left.u\right|_{\partial \Omega}=\varphi, u(\bar{\Omega}) \subset$ $B(p, \mu)$, minimizing $E$ among all such maps, and this map $u$ is a weak solution of (4.6). Moreover, $u$ enjoys the same interior regularity properties as solutions of linear hypoelliptic systems, and if $\partial \Omega$ is smooth and noncharacteristic for $X_{1}, \ldots, X_{m}$, and if $\varphi$ is smooth, one gets the corresponding boundary regularity of $u$.

\section{EXISTENCE OF WEAK SOLUTIONS}

The weighted Sobolev space $M^{1}\left(\Omega, \mathbb{R}^{\nu}\right)$ carries a scalar product with associated norm

$$
\|u\|_{M^{1}}^{2}=\int_{\Omega}|u|^{2} d x+\int_{\Omega}|X u|^{2} d x .
$$

We fix $p \in N$ as in Theorem 1; for $\mu^{\prime}<\min (\pi / 2 \kappa, i(p))$, we put

$$
B_{\mu^{\prime}}:=\left\{u \in M^{1}(\Omega, N) ; \sup _{\Omega} d_{N}(u(x), p) \leq \mu^{\prime}\right\} .
$$

In geodesic normal coordinates with center $p, B_{\mu^{\prime}}$ can be identified with

We also put

$$
\left\{u \in M^{1} \cap L^{\infty}\left(\Omega, \mathbb{R}^{\nu}\right), \sup _{\Omega}|u| \leq \mu^{\prime}\right\} .
$$

$$
B_{\mu^{\prime}}(\Phi):=\left\{u \in B_{\mu^{\prime}}: u-\Phi \in M_{0}^{1}(\Omega, N)\right\} \quad \text { for given } \Phi \in B_{\mu^{\prime}} .
$$

Since $E$ is a Dirichlet integral, $E$ is lower semicontinuous on $B_{\mu^{\prime}}$, i.e. if $\left\{u_{k}\right\} \subset B_{\mu^{\prime}}$ converges weakly to $u$ in $M^{1}\left(\Omega, \mathbb{R}^{\nu}\right)$, then

$$
\lim _{k \rightarrow \infty} \inf E\left(u_{k}\right) \geq E(u) .
$$

The proof can be carried out with the methods of $[\mathrm{G}]$.

Proposition 5.1. Let $\mu^{\prime}<\min (\pi / 2 \kappa, i(p))$. For every $\Phi \in B_{\mu^{\prime}}$, there exists a solution of the variational problem

$$
E(u) \rightarrow \inf _{B_{\mu^{\prime}}(\Phi)} E(v) .
$$


Proof. The above infimum is finite since $E(\Phi)<\infty$. Since $B\left(p, \mu^{\prime}\right)$ is compact and the metric tensor $\left(g_{\alpha \beta}\right)$ is positive definite, there exists $\lambda>0$ with

$$
E(v) \geq \lambda \int_{\Omega}|X v|^{2} d x \quad \text { for all } v \in B_{\mu^{\prime}} .
$$

Consequently, for all $v \in B_{\mu^{\prime}}$,

$$
\|v\|_{M^{1}}^{2} \leq \text { const }\left\{E(v)+\left(\mu^{\prime}\right)^{2}\right\} .
$$

Thus, a minimizing sequence is bounded in $M^{1}\left(\Omega, \mathbb{R}^{\nu}\right)$, and we may assume that it converges weakly to some $u \in B_{\mu^{\prime}}(\Phi)$. By lower semicontinuity of $E$, we must have

$$
E(u)=\inf _{B_{\mu^{\prime}}(\Phi)} E(v),
$$

so that $u$ is the desired minimizer.

q.e.d.

Remark. Using convexity properties of $B\left(p, \mu^{\prime}\right)$ (see $\S 6$ below), one may even construct a minimizing sequence that converges strongly in $M^{1}$, thus bypassing the issue of weak lower semicontinuity of $E$.

In the sequel, we shall need the following chain rule:

Lemma 5.1. Let $u \in C^{2}(\Omega, N), f: N \rightarrow \mathbb{R}, N$ a Riemannian manifold. Then

$$
(-H)(f \circ u)=D^{2} f\left(X_{j} u, X_{j} u\right)+\left\langle(\operatorname{grad} f) \circ u,\left(-H_{N}\right) u\right\rangle_{N},
$$

where $D^{2} f$ is the (Riemannian) Hessian of $f$ and $\langle\cdot, \cdot\rangle_{N}$ the metric of $N$. In particular, if $H_{N} u=0$, then

$$
(-H)(f \circ u)=D^{2} f\left(X_{j} u, X_{j} u\right) .
$$

If in addition, $f$ is strictly convex, with smallest eigenvalue $\lambda>0$ of its Hessian, then

$$
(-H)(f \circ u) \geq \lambda|X u|^{2} .
$$

If we only assume $u \in M^{1}(\Omega, N)$, then under the preceding assumptions, (5.6) holds in the weak sense.

Proof. See the computations in [J1].

q.e.d.

We shall now deduce the following maximum principle:

Lemma 5.2. Under the assumptions of Proposition 5.1, suppose $\Phi \in B_{\mu}$ for some $\mu<\mu^{\prime}$. Then also the minimizer $u$ of Proposition 5.1 is contained in $B_{\mu}$, i.e.

$$
\sup _{\Omega} d_{N}(u(x), p) \leq \mu .
$$

Proof. We consider the function

$$
\begin{gathered}
f: N \rightarrow \mathbb{R}, \\
f(q)=d_{N}^{2}(q, p) .
\end{gathered}
$$

$f$ is smooth on $B\left(p, \mu^{\prime}\right)$, since $\mu<i(p)$, and $f$ is strictly convex on $B\left(p, \mu^{\prime}\right)$, since $\mu<\pi / 2 \kappa$ (see e.g. [J1] for details). This implies that the projection

$$
\pi: B\left(p, \mu^{\prime}\right) \rightarrow B(p, \mu)
$$


along radial geodesics is energy decreasing, i.e.

$$
E(\pi \circ u)<E(u) \quad \text { unless } u(x) \subset B(p, \mu) \quad \text { for almost all } x \in \Omega
$$

(again see [J1]). Since by assumption $\pi \circ \Phi=\Phi$, we have $\pi \circ u \in B_{\mu}(\Phi)$, and (5.8) and the minimizing property of $u$ therefore imply the claim.

q.e.d.

We may now obtain the existence part of the Main Theorem, which we formulate as

Theorem 1. Let $p \in N, \mu<\min (i(p), \pi / 2 \kappa)$, and suppose $\Phi \in M^{1}(\Omega, N)$ satisfies $\Phi(\bar{\Omega}) \subset B(p, \mu)$. Then there exists a weak solution $u$ of

$$
\int_{\Omega} X_{j} u^{\alpha} X_{j} \varphi^{\alpha}-\int_{\Omega} \Gamma_{\beta \gamma}^{\alpha}(u) X_{j} u^{\beta} X_{j} u^{\gamma} \varphi^{\alpha}=0
$$

for all $\varphi \in M_{0}^{1}\left(\Omega, \mathbb{R}^{\nu}\right) \cap L^{\infty}\left(\Omega, \mathbb{R}^{\nu}\right)$,

$$
u-\Phi \in M_{0}^{1}(\Omega, N),
$$

and $u$ satisfies

$$
u(\bar{\Omega}) \subset B(p, \mu) .
$$

Proof. We minimize $E$ in $B_{\mu^{\prime}}(\Phi)$ for some $\mu^{\prime}$ with $\mu<\mu^{\prime}<\min (i(p), \pi / 2 \kappa)$. The solution $u$ exists by Proposition 5.1, and Lemma 5.2 implies $u \in B_{\mu}(\Phi)$, i.e. (5.11). Therefore, $u$ lies in the interior of $B_{\mu^{\prime}}(\Phi)$, and we may perform variations $u+\varepsilon \varphi$ for small enough $|\varepsilon|$ inside $B_{\mu^{\prime}}(\Phi)$ and exploit the minimizing property of $u$ to arrive at (5.9).

q.e.d.

\section{INTERIOR CONTINUITY OF WEAK SOLUTIONS}

Suppose $u$ is a weak solution of $H_{N} u=0(u: M \rightarrow N), f: u(M)(\subset N) \rightarrow \mathbb{R}$ strictly convex, with $\lambda:=$ smallest eigenvalue of $D^{2} f(>0)$. Let

$$
h:=f \circ u .
$$

Then by (5.6)

$$
(-H) h \geq \lambda \sum_{j}\left|X_{j} u\right|^{2} \quad \text { weakly }(=: \lambda e(u)) .
$$

We let $G_{x_{0}}$ be the Green function for $H$ on $\Omega$ with singularity at $x_{0}$.

For $0<\varrho \leq \operatorname{dist}(x, \partial \Omega)$, we let

$$
g_{\varrho}(x):=\min \left(G_{x_{0}}(x)-\varrho^{2}\left|B\left(x_{0}, \varrho\right)\right|^{-1},\left(\frac{\varrho}{2}\right)^{2}\left|B\left(x_{0}, \varrho / 2\right)\right|^{-1}-\varrho^{2}\left|B\left(x_{0}, \varrho\right)\right|^{-1}\right)
$$

and

$$
\Gamma_{\varrho}:=\left\{x: g_{\varrho}(x)=0\right\} .
$$


Then, for $0<\varrho^{\prime}=\frac{\varrho^{\prime \prime}}{2}<\varrho^{\prime \prime}<\operatorname{dist}(x, \partial \Omega)$,

$$
\begin{aligned}
0 & \leq \lambda \int_{0 \leq \varrho \leq \varrho^{\prime \prime}}^{\cup \Gamma_{\varrho}} g_{\varrho^{\prime \prime}} e(u) \\
& \leq \int_{0 \leq \varrho \leq \varrho^{\prime \prime}} g_{\varrho^{\prime \prime}}(-H) h \\
& =-\int_{\varrho^{\prime} \leq \varrho \leq \varrho^{\prime \prime}}^{\cup \Gamma_{\varrho}} X_{j} G_{x_{0}} X_{j} h \\
& =-\int_{0 \leq \varrho \leq \varrho^{\prime \prime}}^{\cup \Gamma_{\varrho}} X_{j} G_{x_{0}} X_{j} h+\int_{\substack{\cup \Gamma_{\varrho} \\
\cup \leq \varrho^{\prime}}} X_{j} G_{x_{0}} X_{j} h
\end{aligned}
$$

(for the existence of these integrals, one uses the mollified Green's function as in Lemma 3.7 and lets the mollification parameter tend to 0$)$

Now let $0<R<\operatorname{dist}(x, \partial \Omega)$ and

$$
R_{i}:=R \cdot 2^{-i} .
$$

Then from (6.2)

$$
\begin{aligned}
& \int_{\substack{0 \leq \varrho \leq R \\
\Gamma_{\varrho}}} G_{x_{0}} e(u) \leq c \sum_{i=0}^{\infty} \int_{0 \leq \varrho \leq R_{i}} g_{R_{i}} e(u) \\
& \leq \frac{c}{\lambda} \sum_{i=0}^{\infty}\left(\mu\left(R_{i}\right)-\mu\left(R_{i+1}\right)\right) \\
& \leq \frac{c}{\lambda} \mu(R) \text {. }
\end{aligned}
$$

In particular,

$$
\int_{B_{H}\left(x_{0}, R\right)} G_{x_{0}} e(u)<\infty,
$$

and given $\delta>0$, for sufficiently large $j$, since $\lambda>0$,

$$
\int_{B_{H}\left(x_{0}, R_{j}\right)} G_{x_{0}} e(u)<\delta .
$$

(For the telescoping trick, see Giaquinta-Giusti [GG] and Giaquinta-Hildebrandt $[\mathrm{GH}]$.

If $u$ is a weak solution of $H_{N} u=0$, then

$$
\int\left\langle X_{j} u, X_{j} \varphi\right\rangle_{N}=0 \text { for all } M_{0}^{1} \text {-sections } \varphi \text { of } u^{-1} T N .
$$

We now choose

$$
\varphi=\eta \cdot G_{y} \cdot(D f) \circ u,
$$

where $f$ is a strictly convex $C^{2}$ function on $u(M), \eta$ is a cut-off function, $\eta \in$ $\operatorname{Lip}\left(B_{H}\left(x_{0}, 2 R_{0}\right)\right), 0 \leq \eta \leq 1, \eta \equiv 1$ on $B_{H}\left(x_{0}, R_{0}\right),\left|X_{j} \eta\right| \leq c_{1} / R_{0}, \operatorname{supp} \eta \subset \subset$ $B_{H}\left(x_{0}, 2 R_{0}\right)$ (see the discussion at the end of $\S 2$ ), and where $G_{y}$ is Green's function on $\Omega$ with singularity this time at $y \in B_{H}\left(x_{0}, R_{0} / 2\right)$, with $B_{H}\left(x_{0}, R_{0}\right) \subset \Omega$. Since $G_{y}$ is unbounded, $\varphi$ as it stands is not an admissible test function, but replacing 
$G_{y}$ by a mollification or a cut-off, one easily sees by an approximation argument using Lemma 3.7 that the subsequent computations are valid. This choice of $\varphi$ is patterned after Hildebrandt-Widman [HW], and so are some of the subsequent estimates. With this choice of $\varphi,(6.6)$ becomes

$$
\begin{aligned}
\int_{B_{H}\left(x_{0}, 2 R_{0}\right)} X_{j} G_{y} X_{j}(\eta f(u))= & -\int_{B_{H}\left(x_{0}, 2 R_{0}\right)} \eta G_{y} D^{2} f\left(X_{j} u, X_{j} u\right) \\
& -\int_{B_{H}\left(x_{0}, 2 R_{0}\right)} G_{y} X_{j} \eta X_{j} f(u) \\
& +\int_{B_{H}\left(x_{0}, 2 R_{0}\right)} f(u) X_{j} G_{y} X_{j} \eta .
\end{aligned}
$$

We write (6.7) as

$$
I=I I+I I I+I V .
$$

Since $\eta=0$ on $\partial B_{H}\left(x_{0}, 2 R_{0}\right)$ and $\eta\left(x_{0}\right)=1$, we have

$$
I=f(u(y)) \text {. }
$$

Since $\eta$ and $G_{y}$ are nonnegative and $f$ is convex,

$$
I I \leq 0 \text {. }
$$

Let $c_{2}:=\sup _{u(M)}|D f|$. Then

$$
\begin{aligned}
I I I & \leq \frac{c_{1} c_{2}}{R_{0}} \int_{B_{H}\left(x_{0}, 2 R_{0}\right) \backslash B_{H}\left(x_{0}, R_{0}\right)} G_{y}\left|X_{j} u\right|_{N} \\
& \leq \frac{c_{1} c_{2}}{R_{0}}\left\{\int_{B_{H}\left(x_{0}, 2 R_{0}\right) \backslash B_{H}\left(x_{0}, R_{0}\right)} G_{y}\right\}^{\frac{1}{2}}\left\{\int_{B_{H}\left(x_{0}, 2 R_{0}\right) \backslash B_{H}\left(x_{0}, R_{0}\right)} G_{y} e(u)\right\}^{\frac{1}{2}} .
\end{aligned}
$$

Now from (3.10)

$$
\int_{B_{H}\left(x_{0}, 2 R_{0}\right) \backslash B_{H}\left(x_{0}, R_{0}\right)} G_{y} \leq c_{3} R_{0}^{2},
$$

and therefore, recalling (6.5), by choosing $R_{0}$ sufficiently small, depending on a given $\varepsilon>0$, we may achieve

$$
I I I \leq \varepsilon,
$$

because the Green functions with singularities at $x_{0}$ and at $y \in B_{H}\left(x_{0}, R_{0} / 2\right)$ are comparable on the annulus $B_{H}\left(x_{0}, 2 R_{0}\right) \backslash B_{H}\left(x_{0}, R_{0}\right)$.

We now estimate IV. We let $u_{R_{0}}$ be the mean value of $u$ on

$$
B_{H}\left(x_{0}, 2 R_{0}\right) \backslash B_{H}\left(x_{0}, R_{0}\right) .
$$

This mean value can be defined with the help of convex geometry on the image of $u$, for example if the image has nonpositive curvature or if the image is contained in a convex ball as described below. Alternatively, one might define it with the help of local coordinates. The different versions will only affect the constants that appear in the Poincaré inequality that will be used in a moment.

We also make the further assumptions that $f$ has a minimum at $u_{R_{0}}$ and that

$$
f\left(u_{R_{0}}\right)=0 .
$$


Then

$$
\begin{aligned}
I V & =\int_{B_{H}\left(x_{0}, 2 R_{0}\right) \backslash B_{H}\left(x_{0}, R_{0}\right)} f(u) X_{j} G_{y} X_{j} \eta \\
& \leq \frac{c_{4}}{\left|B_{H}\left(x_{0}, 2 R_{0}\right)\right|} \int_{B_{H}\left(x_{0}, 2 R_{0}\right) \backslash B_{H}\left(x_{0}, R_{0}\right)} f(u) \quad \text { by }(3.11) \\
& \leq \frac{c_{5}}{\left|B_{H}\left(x_{0}, 2 R_{0}\right)\right|^{\frac{1}{2}}} \sup _{u(M)}|D f|\left\{\int_{B_{H}\left(x_{0}, 2 R_{0}\right) \backslash B_{H}\left(x_{0}, R_{0}\right)} d_{N}\left(u, u_{R_{0}}\right)^{2}\right\}^{\frac{1}{2}} \\
& \leq c_{6}\left\{\frac{R_{0}^{2}}{\left|B_{H}\left(x_{0}, 2 R_{0}\right)\right|} \int_{B_{H}\left(x_{0}, 2 R_{0}\right) \backslash B_{H}\left(x_{0}, R_{0}\right)} e(u)\right\}^{\frac{1}{2}}
\end{aligned}
$$

by the Poincaré inequality (3.8),

where $\sup |D f|$ now has been incorporated into the constant $c_{6}$. (6.5) now can be used as before to obtain

$$
I V \leq \varepsilon
$$

for given $\varepsilon>0$ and sufficiently small $R_{0}$ depending on $\varepsilon$.

Altogether, (6.7)-(6.15) yield

$$
f(u(y)) \leq 2 \varepsilon \quad \text { for all } y \in B\left(x_{0}, \frac{R_{0}}{2}\right),
$$

where $R_{0}$ depends on $\varepsilon$. We may now deduce the interior regularity part of the Main Theorem.

Theorem 2. Let $N$ be a Riemannian manifold, $u: M \rightarrow N$ a bounded weak solution of

$$
H_{N} u=0
$$

Suppose

$$
u(M) \subset B(p, \mu):=\left\{q \in N: d_{N}(p, q) \leq \mu\right\}
$$

for some $p \in N$ with

$$
\mu<\min \left(\frac{\pi}{2 \kappa}, i(p)\right),
$$

where the sectional curvature $K$ of $N$ satisfies $K \leq \kappa^{2}$, and where $i(p)$ is the injectivity radius of $p$. (A ball $B(p, \mu)$ satisfying (6.18) will be called a convex ball.)

Then $u$ is continuous.

Proof. Building upon (6.16), this may be shown by an iteration argument first developed by Wiegner [Wi] and refined by Hildebrandt-Widman [HW], HildebrandtKaul-Widman [HKW], Hildebrandt-Jost-Widman [HJW]. However, the reasoning may be substantially simplified by employing the result of Kendall $[\mathrm{K}]$ that for each $q \in B(p, \mu)$ (where $B(p, \mu)$ is a convex ball), there exists a strictly convex function

$$
f=f_{q}: B(p, \mu) \rightarrow \mathbb{R}
$$

with its unique minimum at $x_{0}$. In fact, inspired by [JäK] we may take 


$$
f_{q}(y)=\frac{1-\cos (\kappa \operatorname{dist}(q, y))}{\cos (\kappa \operatorname{dist}(p, y))}
$$

as shown by Kendall.

Putting $q=u\left(x_{0}\right)$ and $f=f_{u\left(x_{0}\right)}$ in (6.16) immediately yields continuity of $u$ at $x_{0}$, an arbitrary point of $M$.

q.e.d.

Theorem 2 in particular applies in the case where the domain is simply connected and $N$ has nonpositive sectional curvature, because we may lift to universal covers, and on the universal cover of a manifold of nonpositive sectional curvature the injectivity radius is always infinite, so that (6.18) holds for any positive $\mu$.

\section{INTERIOR HÖLDER CONTINUITY}

We return to our decomposition (6.7), (6.8). As in the proof of Theorem 2, by using Kendall's result [K], we may assume that the smallest eigenvalue of $D^{2} f$ is $\geq \lambda>0$. We therefore have, with $y=x_{0}$ and $R$ instead of $R_{0}$,

$$
\lambda \int_{B_{H}\left(x_{0}, R\right)} G_{x_{0}}|X u|^{2} \leq \int_{B_{H}\left(x_{0}, 2 R\right)} \eta G_{x_{0}} D^{2} f\left(X_{j} u, X_{j} u\right) .
$$

We may further assume that $f\left(u\left(x_{0}\right)\right)=0$, so that $I=0$ for $y=x_{0}$ (cf. (6.9)). Using (6.11), (6.14) and Lemma 3.3, we obtain from (6.8)

$$
\int_{B_{H}\left(x_{0}, 2 R\right)} \eta G_{x_{0}} D^{2} f\left(X_{j} u, X_{j} u\right) \leq c_{0} \int_{T_{R}} G_{x_{0}}|X u|^{2},
$$

with the abbreviation

$$
T_{R}:=B_{H}\left(x_{0}, 2 R\right) \backslash B_{H}\left(x_{0}, R\right) .
$$

(7.1) and (7.2) imply

$$
\int_{B_{H}\left(x_{0}, R\right)} G_{x_{0}}|X u|^{2} \leq c_{1} \int_{T_{R}} G_{x_{0}}|X u|^{2} .
$$

We may then use Widman's hole filling, i.e. add

$$
c_{1} \int_{B_{H}\left(x_{0}, R\right)}|X u|^{2} G_{x_{0}}
$$

to both sides of (7.3), to obtain

$$
\int_{B_{H}\left(x_{0}, R\right)}|X u|^{2} G_{x_{0}} \leq \vartheta \int_{B_{H}\left(x_{0}, 2 R\right)}|X u|^{2} G_{x_{0}}
$$

for some $0<\vartheta<1\left(\vartheta=c_{1} /\left(1+c_{1}\right)\right)$. Using the standard iteration method originally introduced by de Giorgi, (7.3) yields

$$
\int_{B_{H}\left(x_{0}, r\right)}|X u|^{2} G_{x_{0}} \leq c_{2} r^{\alpha} R_{0}^{-\alpha} \int_{B_{H}\left(x_{0}, R_{0}\right)}|X u|^{2} G_{x_{0}}
$$

for $0<r<\frac{1}{2} R_{0}$ and some positive $\alpha$. Recalling (6.4), this means that

$$
\int_{B_{H}\left(x_{0}, r\right)}|X u|^{2} G_{x_{0}} \leq c\left(R_{0}\right) r^{\alpha} .
$$

If we then use the estimates (6.11) and (6.14) with $r / 2$ in place of $R_{0}$ and combine them with (7.5), we obtain for $y \in B_{H}\left(x_{0}, r / 4\right)$

$$
f(u(y)) \leq c_{3} r^{\alpha},
$$


and thus Hölder continuity by the properties of $f$. We thus have shown

Theorem 3. Under the assumptions of Theorem 2, $u$ is Hölder continuous in the interior of $\Omega$.

The proof again essentially follows the lines of [HW] and [HKW]. See also [J1].

\section{Regularity uP TO THE BOUNDARY}

We suppose now that the boundary of $\Omega$ is regular in the following sense: $\exists c>0$, $\exists R_{0}>0$ such that for any $x_{0} \in \partial \Omega$, putting $D\left(x_{0}, R\right):=\Omega \cap B_{H}\left(x_{0}, R\right)$, we have

$$
\left|D\left(x_{0}, R\right)\right| \geq C\left|B_{H}\left(x_{0}, R\right)\right| \quad \forall 0<R \leq R_{0} .
$$

Near the boundary, for $R>0$ small enough, we also have the following first Poincaré inequality:

$$
\int_{D\left(x_{0}, R\right)}|\varphi|^{2} d x \leq C R^{2} \int_{D\left(x_{0}, R\right)}|X \varphi|^{2} d x
$$

for any $\varphi \in M_{0}^{1}(\Omega)$. Using the fact that $\left.\varphi\right|_{\partial \Omega \cap B_{H}\left(x_{0}, R\right)}=0$, the proof is classical.

Theorem 4. Let $\varphi \in S^{1}(\partial \Omega, N)$ and assume that $\partial \Omega$ is noncharacteristic for $X_{1}, \ldots, X_{m}$. Suppose that under the assumptions of the preceding theorems, $u$ solves the Dirichlet problem

$$
\begin{aligned}
H_{N} u & =0, \\
\left.u\right|_{\partial \Omega} & =\varphi .
\end{aligned}
$$

Then $u \in S^{\alpha}(\bar{\Omega}, N)$ for some $0<\alpha<1$.

Proof. By Theorem 3, it suffices to prove regularity near the boundary. Let $x_{0} \in$ $\partial \Omega$, and let $f$ be a nonnegative strictly convex function on $B(p, \mu)$ with

$$
f\left(\varphi\left(x_{0}\right)\right)=0
$$

i.e. $\varphi\left(x_{0}\right)$ is the unique minimum of $f$. As explained above, such a function was constructed by Kendall $[\mathrm{K}]$. In order to have a noncharacteristic boundary, we may replace $D\left(x_{0}, R\right)$ by some $\tilde{D}_{R}$ with

$$
D\left(x_{0}, R / 2\right) \subset \tilde{D}_{R} \subset D\left(x_{0}, 3 R / 2\right)
$$

for which $\partial \tilde{D}$ is noncharacteristic for $X_{1}, \ldots, X_{m}$. We then solve the Dirichlet problem

$$
\begin{aligned}
H w_{R} & =0 \text { in } \tilde{D}_{R}, \\
\left.w_{R}\right|_{\partial \tilde{D}_{R}} & =\left.f \circ u\right|_{\partial \tilde{D}_{R}} .
\end{aligned}
$$

The linear regularity theory implies that $w_{R}$ is $S^{\beta}$ near $x_{0}$, since $f \circ \varphi$ is $S^{1}$ on the boundary; more precisely,

$$
w_{R}(y) \leq w_{R}\left(x_{0}\right)+c d_{H}^{\beta}\left(x_{0}, y\right) \quad \text { for } y \in \tilde{D}_{\frac{R}{2}},
$$

for some $0<\beta<1$. (In case $\varphi$ is only assumed to be continuous, for all $\varepsilon>0$ and $R>0$ (sufficiently small), there exists $r$ with

$$
\left.w_{R}(y) \leq w_{R}\left(x_{0}\right)+\varepsilon \quad \text { for all } y \in D\left(x_{0}, r\right) .\right)
$$

By strict convexity of $f$ again, we have

$$
H(f \circ u) \geq \lambda|X u|^{2} \geq 0 \quad \text { for some } \lambda>0 \quad(\text { see (5.6)). }
$$


Thus $H\left(f \circ u-w_{R}\right) \geq 0$, and (8.4)-(8.6) and the maximum principle yield

$$
f(u(y))-f\left(\varphi\left(x_{0}\right)\right) \leq c d_{H}^{\beta}\left(x_{0}, y\right),
$$

hence Hölder continuity of $u$, since $f$ is smooth and strictly convex with a unique minimum at $\varphi\left(x_{0}\right)$.

q.e.d.

Note. If we only assume that $\varphi$ is continuous, we get continuity of $u$ on $\bar{\Omega}$ by using (8.7) in place of (8.6).

\section{Higher REgUlarity}

Theorem 5. Under the assumptions of the main theorem, $u \in S^{1}(\Omega, N)$. If in addition $\varphi \in S^{1, \alpha}(\bar{\Omega}, N)$ and $\partial \Omega$ is sufficiently regular, then $u \in S^{1}(\bar{\Omega}, N)$.

We only outline the proof, since in the preceding sections the reader has already seen sufficiently many instances of adapting known proofs in the elliptic case to the present hypoelliptic case.

Using (3.11), it is not hard to adapt the interior gradient bound of [JK] (see also [J1] and [JY]) to the present situation. Since we do not know yet, however, that $u$ is of class $S^{1}$, we need to combine this estimate with an approximation argument. For that purpose, we approximate the metric $d_{H}$ on $\Omega$ by Riemannian metrics $d_{\varepsilon}$ as described in $\S 2$. We then solve the corresponding Dirichlet problem for the operators $H_{\varepsilon}$ and obtain solutions $u_{\varepsilon}$. These are known to be regular by $[\mathrm{JY}]$, and we get appropriate bounds independently of $\varepsilon$ to allow us to obtain the desired bound for $u=\lim _{\varepsilon \rightarrow 0} u_{\varepsilon}$ (note that the solution of the Dirichlet problem is always unique under our assumptions on the image by [JäK]). In order to get these bounds, one needs to use the analogue of (3.11) for positive $\varepsilon$, with a constant independent of (sufficiently small) $\varepsilon$.

Similarly, the boundary gradient estimate of [JK] (again, see also [J1] and [JY]) extends in an even simpler manner.

Thus, one readily shows Theorem 5 .

Theorem 5 implies that the nonlinear term in our system $H_{N} u=0$ is bounded. One may therefore utilize the regularity theory of linear hypoelliptic PDE (see [X2]) and the standard bootstrap argument to get higher regularity.

This, together with Theorems $1-5$ and the uniqueness result of [JäK] (see [J1] for a somewhat simplified proof), concludes the proof of the Main Theorem.

For a general higher regularity result, we also refer to [XZ].

\section{REFERENCES}

[A1] Al'ber, S.I., On $n$-dimensional problems in the calculus of variations in the large, Sov. Math. Dokl. 5, (1964), 700-704 MR 29:2685

[A2] Al'ber, S.I., Spaces of mappings into a manifold with negative curvature, Sov. Math. Dokl. 9, (1967), 6-9 MR 37:5817

[B] Bony, J.M., Principe du maximum, inégalité de Harnack et unicité du problème de Cauchy pour les opérateurs elliptiques dégénérés, Ann. Inst. Fourier 19 (1969), 227-304 MR 41:7486

[BM] Biroli, M., Mosco, U., Formes de Dirichlet et estimations structurelles dans les milieux discontinues, C. R. Acad. Sci. Paris 313 (1991), 593-598, and: A Saint-Venant type principle for Dirichlet forms on discontinuous media, Annali Mat. Pura Appl. (4) 169 (1995), 125-181 MR 93c:35028; MR 97b:35082 
[ES] Eells, J., and Sampson, J., Harmonic mappings of Riemannian manifolds, Am. J. Math. 85 (1964), 109-160 MR 29:1603

[FP] Fefferman, C., and Phong, D.H., Subelliptic eigenvalue problems, Proceedings of the Conference on Harmonic Analysis, in Honor of A. Zygmund, Wadsworth Math. Series, 1981, 590-606 MR 86c:35112

[G] Giaquinta, M., Multiple integrals in the calculus of variations and nonlinear elliptic systems, Princeton Univ. Press (1983) MR 86b:49003

[GG] Giaquinta, M., and Giusti, E., On the regularity of the minima of variational integrals, Acta Math. 148 (1982), 31-46 MR 84b:58034

[GH] Giaquinta, M., and Hildebrandt, S., A priori estimates for harmonic mappings, Journ. reine angew. Math. 336 (1982), 123-164 MR 84b:58035

[H] Hörmander, L., Hypoelliptic second order differential equations, Acta Math. 119 (1967), 141-171 MR 36:5526

[HJW] Hildebrandt, S., Jost, J., Widman, K.O., Harmonic mappings and minimal submanifolds, Inv. Math. 62 (1980), 269-298 MR 82d:58025

[HKW] Hildebrandt, S., Kaul H., Widman K., An existence theorem for harmonic mappings of Riemannian manifolds, Acta Math. 138 (1977), 1-16 MR 55:6478

[Hm] Hamilton, R., Harmonic maps of manifolds with boundary, Springer LNM 471 (1975) MR 58:2872

[HW] Hildebrandt, S., Widman K., On the Hölder continuity of weak solutions of quasilinear elliptic systems of second order, Ann. Sc. Norm. Sup. Pisa (IV) 4 (1977), 145-178 MR 56: 16140

[J1] Jost, J., Harmonic mappings between Riemannian manifolds. Proc. Centre Math. Anal., ANU-Press, Vol. 4 (1983) MR 86b:58030

[J2] Jost, J., Equilibrium maps between metric spaces, Calc. Var. Partial Differential Equations 2 (1994), 173-204 MR 98a:58049

[J3] Jost, J., Convex functionals and generalized harmonic maps into spaces of nonpositive curvature, Comm. Math. Helv. 70 (1995), 659-673 MR 96j:58043

[J4] Jost, J., Generalized harmonic maps between metric spaces, in: Geometric Analysis and the Calculus of Variations for Stefan Hildebrandt (J.Jost, ed.), Internat. Press, Cambridge, MA, 1996, 143-174 CMP 97:12

[J5] Jost, J., Generalized Dirichlet forms and harmonic maps, Calc. Var. Partial Differential Equations 5 (1997), 1-19. CMP 97:05

[JäK] Jäger, W., and Kaul, H., Uniqueness and stability of harmonic maps and their Jacobi fields, Manuscripta Math. 28 (1979), 269-291 MR 80j:58030

[Je] Jerison, D., The Poincaré inequality for vector fields satisfying Hörmander's condition, Duke Math. J. 53 (1986), 503-523 MR 87i:35027

[JeS] Jerison, D., Sánchez-Calle, A., Subelliptic second-order differential operators, Lecture Notes in Math. 1277, 46-77 MR 89b:35021

[JK] Jost, J., and H. Karcher, Geometrische Methoden zur Gewinnung von a-priori-Schranken für harmonische Abbildungen, Man. math. 40 (1982), 27-77 MR 84e:58023

[JY] Jost, J., Yau, S.T., A nonlinear elliptic system for maps from Hermitian to Riemannian manifolds and rigidy theorems in Hermitian geometry, Acta Math. 170 (1993), 221-254; 173 (1994), 307. MR 94g:58053; MR 95h:58040

[K] Kendall, W., Convexity and the hemisphere, J. London Math. Soc. (2) 43 (1991), 567-576, and: Probability, convexity, and harmonic maps with small image I: uniqueness and fine existence, Proc. London Math. Soc. (3) 61 (1990), 371-406 MR 92h:58021; MR 91g:58062

[NSW] Nagel, A., Stein, E.M., and Wainger, S., Balls and metrics defined by vector fields I, basic properties, Acta Math. 155 (1985), 103-147 MR 86k:46069

[RS] Rothschild, L., Stein, E.M., Hypoelliptic operators and nilpotent Lie groups, Acta Math. 137 (1977), 247-320 MR 55:9171

[S] Sánchez-Calle, A., Fundamental solutions and geometry of the sum of squares of vector fields, Invent. Math. 78 (1984), 143-160 MR 86e:58078

[Wi] Wiegner, M., A priori Schranken für Lösungen gewisser elliptischer Systeme, Manuscripta Math. 18 (1976), 279-297 MR 53:6093

[X1] Xu, C.J., Subelliptic variational problems, Bull. Soc. Math. France 118 (1990), 147-169 MR 92b: 49008 
[X2] Xu, C.J., Regularity for quasilinear second order subelliptic equations, Comm. Pure Appl. Math., 1992, 77-96 MR 93b:35042

[XZ] Xu, C.J., Zuily, C., Higher interior regularity for quasilinear subelliptic systems, Calc. Var. Partial Differential Equations 5 (1997), 323-343. CMP 97:13

Institut für Mathematik, Ruhr-Universität Bochum, 44780 Bochum, Germany

Current address: Max-Planck-Institut für Mathematik in den Naturwissenschaften, Inselstr. 22-26, D-04103 Leipzig, Germany

E-mail address: jost@mis.mpg.de

Department of Mathematics, Wuhan University, 430072 Wuhan, China

Current address: Université de Rouen, Analyse et modèles stochastiques, UPRAS-A 6085, UFR des Sciences, Mathématiques, F-76821 Mont St. Aignan, France

E-mail address: chao-jiang.xu@univ-rouen.fr 